Hemianopia in Untreated Secondary Syphilis

\title{
HEMIANOPIA AS THE SOLE CLINICAL FEATURE IN UNTREATED SECONDARY SYPHILIS
}

\author{
BY \\ Al.an W. S. Sichel, M.D.(Edin.), D.O.(Oxon.), D.O.M.S.(Eng.) \\ AND \\ A. Reith Fraser, M.D.(Aberd.) \\ CAPE TOWN, SOUTH AFRICA
}

IT is well known that cerebro-spinal implication in syphilis is an early manifestation and not, as was previously taught, a "tertiary" phenomenon. Its obvious clinical exhibition, however, is not always apparent. Many workers have shown that the majority of cases of syphilis in the secondary stage, and many in the primary stage, show definite biological and cytological evidences of intrathecal involvement.

It is highly probable that generalization of syphilitic infection has occurred before the chancre appears. The experimental work of Metchnikoff and Roux, Neisser, Brandes, and others has proved conclusively that the localization' of the spironema does not exceed 24 hours. Any local evidence of reaction to the invader does not appear for an average period of 21 days. During this time lymphatic and vascular spread of the parasite has been taking place, but at what rate we can only surmise. We know, however, that when local reaction occurs and the chancre appears at the point of entry, generalization has advanced so far that local destruction or excision of the chancre is unable to modify or abort the disease. Already all tissues are equally accessible to the parasite. It follows then that the first tissue to be attacked is that which affords the most favourable conditions for proliferation and growth of the spironema. The first tissue to show clinical evidence of such attack is that which possesses the lowest powers of resistance, which is so situated that it receives the greatest flood of spironemata, and which is most susceptible to damage and capable of showing clinical evidence of such damage.

There is a good deal of evidence to show that the spironema in the new host flourishes best in the tissue from which it originally was derived. This conception has led to the differentiation of spironemata into two distinct strains-the one dermotrophic and the other neurotrophic. It has been stated that these strains can be differentiated morphologically, but this is not generally accepted. Such differentiation into strains, therefore, is based on clinical observations.

In the cerebro-spinal axis the spironema pallidum has no 
exclusive site of attack. This we would take for granted were we to consider the all-important fact that the mode of dissemination of the spironema is one of vascular and lymphatic spread. Moreover, these structures are subjected to grave attack during the process of this dissemination. Syphilitic septicaemia is exhibited by pyrexia, headache, malaise, and so on frequently long before the surface evidence of so-called secondary syphilis is apparent. We have no reason to expect that the cerebro-spinal axis should escape during this period of septicaemia. We should, therefore, expect the disease in the central nervous system to be essentially one of lympharteritis, and this histological study helps to confirm.

It is the fact, however, that a dominating cerebral manifestation in the early stage of syphilis is an occurrence of much rarity. Even in cases where the infecting strain of spironema is believed to be neurotrophic the earlier manifestations, with the exception of serological and cytological changes, usually are absent. This may be due to the fact that disease of the lymphatics rarely betrays itself by clinical symptoms. Where the strain is not considered neurotrophic sui generis, any cerebro-spinal manifestations usually occur while the cutaneous lesions are still in process of evolution or resolution.

In these days of salvarsan therapy of syphilis, meningeal irritation and the so-called "neuro-recidives" have received much attention. These curious manifestations of neuro-syphilis vary from a mild clinical variety including headache, insomnia, tinnitus and vertigo, through the characteristic implication of individual cranial nerves, especially the second and eighth, to a severe type which exhibits such symptoms as mental confusion, epileptic fits, dementia and even coma. They are partly meningo-lymphovascular and partly parenchymatous. The generally accepted explanation is that they are precipitated by interference with antibody supply resulting from inefficient massive-dose salvarsan therapy. Spironemata are destroyed very suddenly and an intense local inflammatory reaction occurs in the immediate neighbourhood. Endotoxins are released which attack the brain or meninges producing a neuro-relapse. By pushing salvarsan further the remaining circulating spironemata are dealt with and the lesion disappears. Such neuro-recidives are by no means rare, but their incidence is always subsequent to the administration of salvarsan or its derivatives or substitutes. Such an isolated lesion must not be confused with a neuro-syphilide occurring in the early stage of syphilis in the absence of salvarsan therapy. Such a neuro-syphilide is uncommon. For this reason we consider the presentation of the following case justified, particularly on account of the fact that the ocular lesion was the sole clinical 
evidence of systemic disease at a time when one would have been expected to search the skin and mucous membranes for signs of luetic activity.

In syphilitic lesions of the cerebro-spinal axis we recognize two distinct classes. The first is a meningo-lympho-vascular type which includes gummatous and endarteritic processes. Pressure symptoms from large gummata invading the brain and cord are well known. These arise from non-nervous tissues. Diffuse gummatous infiltrations may occur in the meninges of bloodvessels of the cerebro-spinal axis. Endarteritis culminates frequently in thrombosis of the affected artery resulting in an infarct in the brain or cord followed by corresponding sclerosis. The second class is one of parenchymatous nervous disease characterized by primary degeneration of specialized nerve cells and nerve tracts. For this class of disease the direct action of syphilitic toxin upon the nervous structures is responsible. Sclerotic overgrowth of the neuroglia in the affected areas may follow primary degenerative lesions, quite independent of any gross meningeal, lymphatic or vascular lesion. Examples of this group are found in dementia paralytica, tabes dorsalis, Eirb's syphilitic paraplegia, and certain forms of primary optic atrophy. The important difference between these two groups lies in the prognosis. In the first class prognosis is good, whereas in the second class prognosis is aiways relatively bad-at the most we can hope to arrest the process, but we cannot attempt to produce anatomical repair.

Hemianopia is by no means a rare manifestation of syphilitic meningeal affection. It may be found in those cases where the visual tracts are affected, the lesion occurring anywhere between the chiasma and the occipital lobes. This symptom, however, is usually a late one, due to endarteritis, perivascular infiltration and gummatous changes.

In specific basilar meningitis the cranial nerves are rarely individually affected. As a rule the several cranial nerves situated in close relationship anatomically are involved simultaneously. Oscillating hemianopia (hemianopia fugax) has been described as being characteristic of basilar syphilis.

The optic nerve is usually secondarily affected--that is to say, there is no primary affection of the nerve substance itself, but changes are produced by extension from the meninges. Certain forms of primary optic atrophy, however, belong to the toxic parenchymatous group. By far the most common lesion is compression by gummatous proliferations or infiltrating meningitis. Such changes as choked disk, optic neuritis, irregularities in the field of vision, scotomata, optic atrophy, and so on are well recognized as being luetic stigmata. 


\section{Report of a case}

The patient, H.P., an unmarried European of 29 years of age, developed a hard chancre on the left side of the glans penis in the latter part of October, 1921, in East Africa (Mombassa). This sore healed without treatment of any kind in eight weeks' time, and, being a long distance from medical aid, he received no treatment. He had no other evidence of syphilitic infection. Being an educated man, however, he was uneasy concerning his infection, and, arranging for leave forthwith, came to Cape Town on February 14, 1922. He stated that the sore was his only symptom until, coming along the African coast, he thought his vision was not so good as it had been when he left Mombassa. By direct questioning (A.W.S.S.) it was ascertained that he first noticed a "film" before his eyes towards the right side not more than two weeks previously. When reading he had to turn his head to the right in order to see words at the ends of the printed lines. There was no other complaint, ophthalmic or otherwise. A recent large indurated scar was apparent on the glans penis. Adenopathy was general. There was no skin or mucous membrane lesion of any kind, old or recent. Examination of the central nervous system revealed no abnormality whatever apart from the eye condition. The serum Wassermann Reaction was ++++ (four plus), the serum fixing the maximum number of M.H.D.'s of complement. The patient declined to have his spinal fluid examined.

On ophthalmic examination $(17 / 2 j 22)$ the following data were obtained :-

Visual Acuity.-R.V. 5/36. L.V. 5/5 partly : under cycloplegia (homatropin and cocain) R. $V$. with $+3 \mathrm{D}$. sph.1D. cyl. ax. $20 \% /=/ 5 / 24$. L.V. with $+0.5 \mathrm{D}$ sph. $=5 / 5$ partly.

Accommodation.-Punctum proximum : R. $=17 \mathrm{~cm} . \mathrm{L} .=14 \mathrm{~cm}$.

Muscle Balance.-Orthophoria at 4 metres. $12^{\circ}$ exophoria (varying) at $33 \mathrm{~cm}$.

Tension.- R. and L. normal.

Pupils.-These were regular in contour, equal in size, and reacted briskly to light, direct and consensual, and to accommodation-convergence. Wernicke's hemiopic reaction could not be elicited.

Fundi.-There were no pathological changes present.

Ocular Excursions.- These were unimpaired in the six cardinal directions. No diplopia was elicited by the candle test, and there was no nystagmus.

Lids.-Movements were full and strong, and there was no ptosis. The left palpebral aperture was slightly smaller than that on the right side.

Corneal Sensibility.--This was not impaired on either side. 
Fields of Vision.--There were no central colour scotomata. Perimetric charts (Fig. 1) showed complete loss of vision in the right half of each field, the line of demarcation passing to the right of the fixation point.

LEFT.

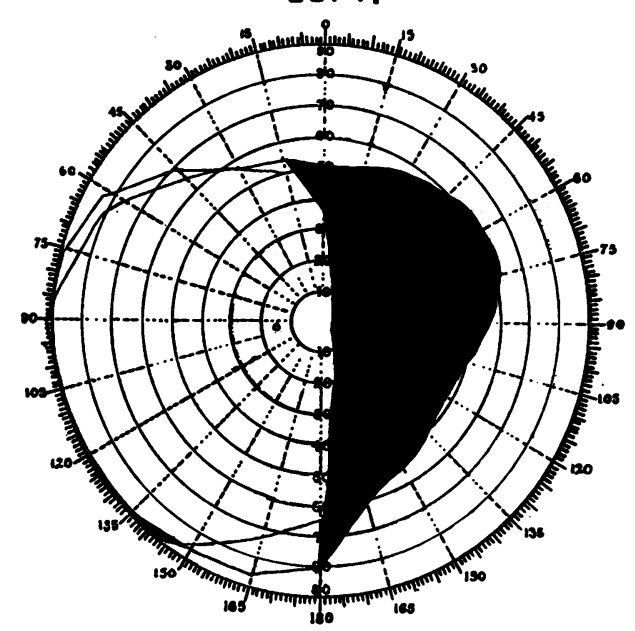

RIQHT.

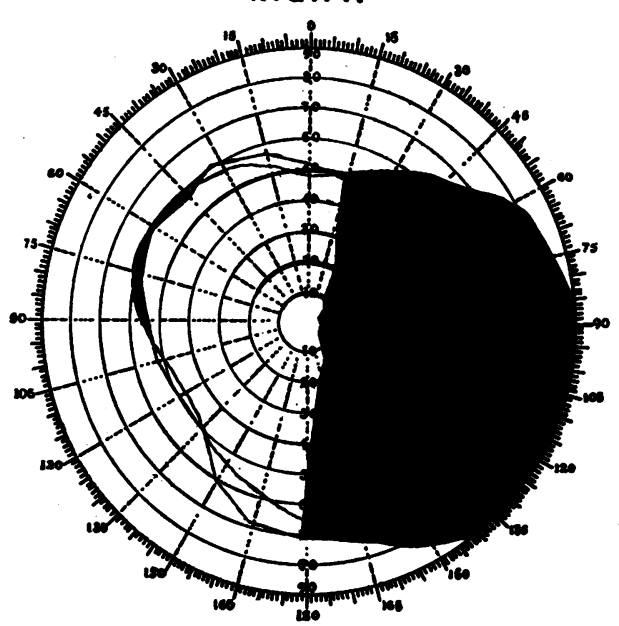

FIG. 1.

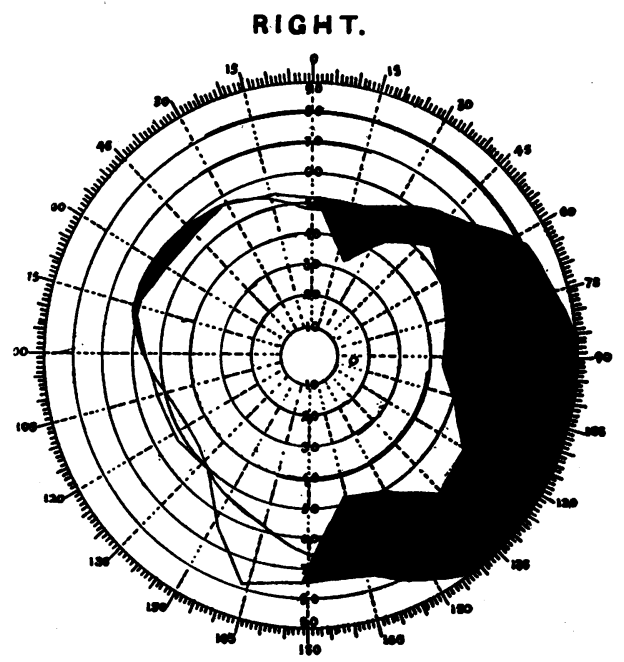

FIG. 2.

Treatment was instituted immediately, but on account of the fact that the patient lived some thirty miles out of Cape Town, it was found impossible to arrange the therapy which one would have liked. Moreover, his leave was limited, and specific arsenobenzene treatment could not be pushed to its furthest limits. He 
received, however, 1.95 grammes novarsenobenzol intravenously in four weeks, spread over ten doses (14/2/22 till 17/3/22). Larger doses were not considered indicated on account of the risk of damaging nervous tissue which had already proved itself to be susceptible to attack. Mercury was administered by mouth in the form of pil. Hg. protoiodide, gr. 1/3, t.i.d. This he was instructed to continue for three years, 21 days per month.

Precisely one month after the previous perimetric reading, the fields of vision indicated a recession of the blind area to approximately the $50^{\circ}$ circle : i.e., a relatively complete recovery in the left eye and more than half the full extent of the normal field in the right eye. (Fig. 2.)

No other systemic or nervous symptoms developed in the meantime. On account of force of circumstances he returned to East Africa on 18/3/22. The publication of this note has been delayed in the hope that the patient might reappear in Cape Town for further observation and treatment, but this hope has not been realized.

\section{Comment}

From the ophthalmic standpoint the case resolved itself into one of a right-sided homonymous hemianopia as the sole pathological feature. The complete absence of systemic syphilitic manifestations is particularly striking. The slight difference in size between the palpebral apertures may have been congenital or possibly due to sympathetic irritation though proptosis was absent.

The lesion present would be left-sided, and situated at any point along the optic tract from the chiasma to the sub-cortical and cortical centres. In the absence of other clinical signs there was no means of accurately localizing the point of interference with visual perception excepting the significance of the hemiopic pupil reaction which, in this instance being absent, would suggest that the lesion was not situated in the optic tract itself.

\section{ANNOTATION}

\section{Miners' Nystagmus: the question of compensation}

In an annotation the Lancet (August 11, 1923) draws attention to the Second Report of the Miners' Nystagmus Committee of the Medical Research Council, which discloses what appears to be a serious flaw in the Workmen's Compensation Act. After detailing the causes of the great and increasing relative prevalence of compensated inçapacity from miners' nystagmus as compared 